\title{
Effects of Bepridil on Stretch-Activated BKca Channels and Stretch- Induced Extrasystoles in Isolated Chick Hearts
}

\author{
H. JIN ${ }^{1}$, G. IRIBE ${ }^{2}$, K. NARUSE ${ }^{2}$ \\ ${ }^{1}$ Department of Pharmacy, The Affiliated Hospital of Yanbian University, Yanji City, JiLin \\ Province, China, ${ }^{2}$ Cardiovascular Physiology, Okayama University Graduate School of Medicine, \\ Dentistry and Pharmaceutical Sciences, Shikata-cho, Okayama, Japan
}

Received February 3, 2016

Accepted November 25, 2016

On-line February 28, 2017

\begin{abstract}
Summary
Various types of mechanosensitive ion channels, including cationic stretch-activated channels $\left(\mathrm{SAC}_{\mathrm{NS}}\right)$ and stretch-activated BKca (SAKca) channels, modulate heart rhythm. Bepridil has been used as an antiarrhythmic drug with multiple pharmacological effects; however, whether it is effective for mechanically induced arrhythmia has not been well investigated. To test the effects of Bepridil on SAKca channels activity, cultured chick embryonic ventricular myocytes were used for singlechannel recordings. Bepridil significantly reduced the open probability of the SAKca channel $\left(P_{0}\right)$. Next, to test the effects of bepridil on stretch-induced extrasystoles (SIE), we used an isolated 2-week-old Langendorff-perfused chick heart. The left ventricle (LV) volume was rapidly changed, and the probability of SIE was calculated in the presence and absence of bepridil, and the effect of the drug was compared with that of Gadolinium $\left(\mathrm{Gd}^{3+}\right)$. Bepridil decreased the probability of SIE despite its suppressive effects on SAKca channel activity. The effects of $\mathrm{Gd}^{3+}$, which blocks both SAKca and SAC ${ }_{\mathrm{NS}}$, on the probability of SIE were the same as those of bepridil. Our results suggest that bepridil blocks not only SAKca channels but possibly also blocks $\mathrm{SAC}_{\mathrm{NS}}$, and thus decreases the stretch-induced cation influx (stabilizing membrane potential) to compensate and override the effects of the decrease in outward SAKca current (destabilizing membrane potential).
\end{abstract}

\section{Key words}

Stretch-activated channels - Arrhythmia - SAKca channels • Bepridil

\section{Corresponding authors}

H. Jin, Department of Pharmacy, The Affiliated Hospital of YanBian University, No.1327 JuZi Street, YanJi City, JiLin Province, China. Fax: +86-433-2660168. E-mail: kimflower1988@163.com.

G. Iribe, Cardiovascular Physiology, Okayama University Graduate School of Medicine, Dentistry and Pharmaceutical Sciences, 2-5-1, Shikata-cho, Kita-ku, Okayama 700-8558, Japan. Fax: +81-86-235-7430. E-mail: iribe@md.okayama-u.ac.jp

\section{Introduction}

Mechanical stimuli modulate cardiac function at various levels. For instance, differentiation of Purkinje fibers from myocytes during embryogenesis is affected by mechanical stimuli via endothelin (Hall et al. 2004). In mature heart, mechanical stimuli affect the electrophysiological properties of the heart and sometimes cause arrhythmic events (mechanoelectric feedback; MEF) (Kohl et al. 1999, Lab et al. 1996). For instance, inappropriate placement of a transvenous right ventricular electrode or a pulmonary artery catheter sometimes produces an excessive intraventricular catheter loop, and physically stimulates the ventricular wall to cause catheter-induced arrhythmia (Voukydis et al. 1974). Mediastinal chest drainage tubes, which are inserted after cardiac surgery as a standard practice, could result in physical arrhythmogenic stimulation during the postoperative period (Lim-Levy et al. 1986). One of the most critical examples of MEF is commotio cordis (fatal arrhythmia caused by a blunt precordial impact without 
structural damage), which was first reported 130 years ago (Nesbitt et al. 2001). A precordial impact not only causes arrhythmia but can also effectively terminate ventricular tachycardia and fibrillation (Pellis et al. 2010).

This kind of mechanically induced modulation of heart rhythm is mediated by various types of mechanosensitive channels. For instance, in a Langendorff-perfused rabbit heart, stretch-induced atrial fibrillation could be suppressed by Grammostola spatulata mechanotoxin 4 (GsMTx4), a specific blocker of cationic stretch-activated channels $\left(\mathrm{SAC}_{\mathrm{NS}}\right.$ ) (Bode et al. 2001). We have also shown that the incidence of leftventricular-wall stretch-induced extrasystole (SIE) was reduced by GsMTx 4 and enhanced by Iberiotoxin (IbTx), a sarcolemmal stretch-activated BKca (SAKca) channel blocker, in isolated Langendorff-perfused chick hearts (Iribe et al. 2011).

The SAKca channel was originally cloned from cultured chick embryo ventricular myocytes as a splicing variant of the BKca channel and includes a stress axisregulated exon (STREX) sequence, which is responsible for its stretch sensitivity (Naruse et al. 2009). Interestingly, STREX variants of BKca channels in chicks and humans share a short amino acid residue sequence (ERA) and show stretch sensitivity. On the other hand, STREX variants of these channels in mice and rabbits do not have the ERA sequence and do not show stretch sensitivity (Naruse et al. 2009). Therefore, chick heart could be a good model to estimate mechanically induced changes in cardiac electrophysiology in the human heart.

Bepridil was originally developed as an antianginal drug due to its vasodilating and negative inotropic effects (Cosnier et al. 1977, Singh 1991). It is also known as an antiarrhythmic drug (Iijima et al. 2010, Yamashita et al. 2009) with multiple pharmacological effects including inhibition of $\mathrm{Na}^{+}, \mathrm{K}^{+}$, and $\mathrm{Ca}^{2+}$ currents (Cohen et al. 1992, Gill et al. 1992, Wang et al. 1999). Despite its multiple effects on myocardial electrophysiology, whether or not it affects mechanosensitive channels has not been thoroughly investigated. In this study, we investigated the effects of bepridil on single SAKca channel current in cultured chick ventricular myocytes and also estimated the effects on $\mathrm{SAC}_{\mathrm{NS}}$ of drug-induced changes in SIE properties using isolated Langendorff-perfused chick hearts with a computer-controlled left ventricle (LV) wall stretch system.

\section{Methods}

All experiments were performed according to the Guiding Principles for the Care and Use of Animals approved by the Council of the Physiological Society of Japan. The study protocol was approved by the Animal Subjects Committee of Okayama University Graduate School of Medicine, Dentistry, and Pharmaceutical Sciences.

\section{Cell Culture}

Cultured chick ventricular myocytes were prepared as described elsewhere (Kawakubo et al. 1999, Tang et al. 2003). In brief, ventricles were dissected from 10- to 12-day-old White Leghorn embryos under sterile conditions. The ventricles were minced in $\mathrm{Ca}^{2+}-$ and $\mathrm{Mg}^{2+}$ - free saline, and then exposed to saline containing $2 \mathrm{mg} / \mathrm{ml}$ collagenase type IA (Sigma Chemical) for $10 \mathrm{~min}$ at $37^{\circ} \mathrm{C}$ to prepare cell suspensions. Cells were cultured in Dulbecco's modified Eagle's medium (DMEM; GIBCO BRL) supplemented with horse serum $(10 \% \mathrm{vol} / \mathrm{vol})$ and chick embryo extract $(2 \% \mathrm{vol} / \mathrm{vol})$. Cells from 3 to 14 days of culturing were used for experiments.

\section{Single-Channel Recording}

Electrophysiological single-channel experiments were performed in the excised inside-out configuration of the patch-clamp technique. Patch electrodes were fabricated from disposable micropipettes (GD-1.5; Narishige Scientific Instrument Lab, Japan) in two stages on a vertical electrode puller (PP-83; Narishige Scientific Instrument $\mathrm{Lab}$ ). The tips of the patch pipettes were firepolished on a microforge (MG-83; Narishige Scientific Instrument Lab). In order to get single channel recording glass pipettes with an electrode resistance of 5-15 M $\Omega$ were used. Currents were amplified using an Axopatch 200B patch-clamp amplifier (Axon Instruments, CA) sampled at $8-10 \mathrm{kHz}$ and filtered at $2 \mathrm{kHz}$ via a 4-pore low-pass Bessel filter. Data acquisition and analysis were performed using the software pClamp 9.0 (Axon Instruments). In most cases the probability of the channel being open $\left(P_{\mathrm{O}}\right)$ was simply calculated from the total time spent in the open state divided by the total time of the recording for the patches containing a single channel. When multiple channels were present in the patch, $P_{\mathrm{O}}$ was calculated from the equation

$$
P_{\mathrm{O}}(\%)=\left(1-P_{\mathrm{C}}^{1 / \mathrm{N}}\right) \times 100,
$$


where $P_{\mathrm{C}}$ is the fraction of the closed state, and $\mathrm{N}$ is the number of channels in the patch. Continuous recording of 2,000 ms were used to estimate $P_{\mathrm{O}}$ values. Mechanical stretch in the patch was created by applying negative pressure in the pipette with a pneumatic transducer tester (DPM-1B; Bio-Tek Instruments) connected to a pipette holder. Single-channel recordings were made at room temperature $\left(22-25^{\circ} \mathrm{C}\right)$. The pipette solution facing the extracellular surface of the patch contained $145 \mathrm{mM} \mathrm{KCl}$, $10 \mathrm{mM}$ HEPES, and $10 \mathrm{mM}$ glucose adjusted to $\mathrm{pH} 7.4$ with $\mathrm{KOH}$. The bath solution facing the cytoplasmic surface of the patch contained the same solution with $1 \mathrm{mM}$ EGTA and $0.94 \mathrm{mM} \mathrm{CaCl}_{2}$ to achieve $1 \mu \mathrm{M}$ free $\mathrm{Ca}^{2+}$. These data was calculated using program EQCAL (Biosoft, MO) with the stability constants from reference (Owen 1976). To confirm that the channel in the patch is SAKca, single channel conductance was checked in six cells as follows. The single channel currents traces were obtained in symmetric $145 \mathrm{mM} \mathrm{K}^{+}$solution with $1 \mu \mathrm{M}$ free $\mathrm{Ca}^{2+}$ at $-60,-30,+30$ and $+60 \mathrm{mV}$, then single channel conductances were calculated according to current-voltage relationships.

\section{Langendorff-perfused heart preparation}

Langendorff-perfused hearts were prepared as described elsewhere (Iribe et al. 2011). In brief, 2-week-old chicks (body weight 90-120 g) were heavily anesthetized with ether. After paramedian sternotomy, the heart was rapidly excised from chest and washed in cold normal Tyrode (NT) solution $(136 \mathrm{mmol} / \mathrm{l} \mathrm{NaCl}$, $5.4 \mathrm{mmol} / \mathrm{l} \mathrm{KCl}, 1 \mathrm{mmol} / 1 \mathrm{MgCl}, 0.3 \mathrm{mmol} / 1 \mathrm{NaH}_{2} \mathrm{PO}_{4}$, $1.8 \mathrm{mmol} / 1 \mathrm{CaCl}_{2}, 10 \mathrm{mmol} / \mathrm{l}$ glucose, and $5 \mathrm{mmol} / \mathrm{l}$ HEPES; pH 7.4 adjusted with $\mathrm{NaOH}$ ). The pericardium was then removed. The aorta was cannulated and two major branches from the arch of the aorta were ligated. The heart was then perfused with NT solution at a constant flow rate of $12 \mathrm{ml} / \mathrm{min}$ using a flow roller pump system. The left atrium appendage was opened and a thin latex balloon with an unstressed volume of $150 \mu \mathrm{l}$ was fitted into the LV chamber. The balloon was connected to our custom-made LV volume (LVV) changer and a pressure transducer; the circuit was primed with water. The LVV changer comprised a $0.5-\mathrm{ml}$ syringe connected to a rapid microstepping motor (Fig. 1). The stepper was controlled by custom software written in LabView (National Instruments, Austin, TX). Electrocardiogram (ECG) and LV pressure (LVP) were continuously monitored. The data were digitized and acquired online at $500 \mathrm{~Hz}$ by custom LabView software and a fast analogue-to-digital/digital-to- analogue interface (NIDAQCard-6062E; National Instruments). The temperature of the heart was maintained at $37^{\circ} \mathrm{C}$, and the perfusate was oxygenated throughout the experiment.

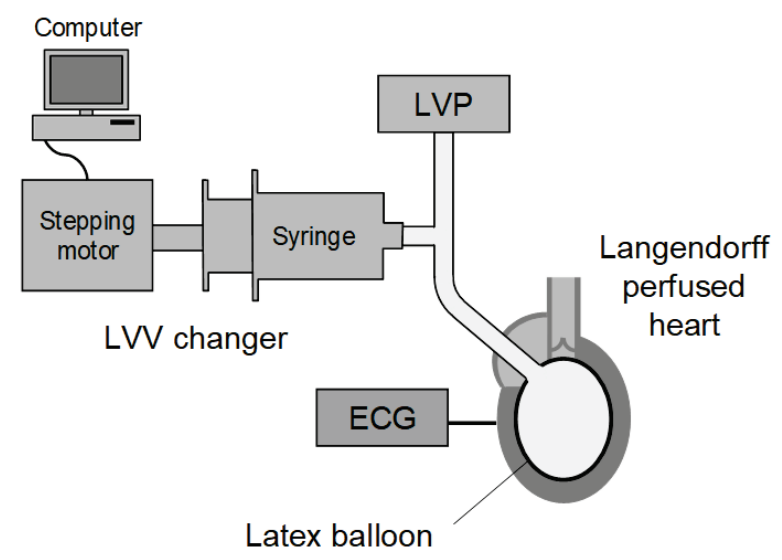

Fig. 1. Schematic overview of the left ventricular (LV) wall stretch system for stretch-induced extrasystole experiments. LV volume (LVV) was controlled by changing the volume of a latex balloon inserted into the LV. LV pressure (LVP) and electrocardiogram (ECG) were monitored.

\section{SIE protocol}

Initial LVV (Vi) was adjusted such that the diastolic LVP was $10 \mathrm{mmHg}(35-45 \mu \mathrm{l})$. After obtaining a stable and regular beating state under sinus rhythm, a transient LV wall stretch pulse was applied by inflating and deflating the LV balloon with $40 \%$ of Vi $(40 \mathrm{~ms}$ inflation, $10 \mathrm{~ms}$ plateau, and $40 \mathrm{~ms}$ deflation). The upstroke of LVP was used as a trigger to identify the start of a cardiac cycle, and an approximately 200-ms delay was inserted before the pulse. The delay time was adjusted to ensure that the pulse was applied after LVP returned to the diastolic pressure. Stretch was repeated 20 times, and the percentage of SIE occurrence was calculated to give the probability of SIE.

\section{Statistics}

All the values are presented as mean \pm standard error of the mean (SEM). Statistical assessment was performed using two-way ANOVA. A $P$ value of less than 0.05 was considered to indicate a significant difference between means.

\section{Results}

The slope conductance of the SAKca channels was $262.8 \pm 10.9 \mathrm{pS}(\mathrm{n}=6)$, which was consistent with those reported in previous studies (Kawakubo et al. 1999, 
Tang et al. 2003).

Figure 2 shows dose-dependent effect of bepridil on single SAKca channel activities in symmetric $145 \mathrm{mM}$ $\mathrm{K}^{+}$solutions at $+30 \mathrm{mV}$. In the absence of bepridil, $P_{\mathrm{O}}$ was almost fully opened $\left(P_{\mathrm{O}}=96.24 \pm 0.58 \%, \mathrm{n}=13\right)$ at a membrane potential of $+30 \mathrm{mV}$ (panel A, top). This $P_{\mathrm{O}}$ value is reasonable for SAKca channels with a free $\mathrm{Ca}^{2+}$ concentration of $1 \mu \mathrm{M}$ (Kawakubo et al. 1999, Tang et al. 2003). In the presence of bepridil, the $P_{\mathrm{O}}$ value decreases in a dose-dependent manner and almost completely blocked with $20 \mu \mathrm{M}$ bepridil (panel A, bottom). The concentration-dependent inhibition of bepridil on the channel activities is summarized and plotted in panel B. We fitted the data with the following Hill equation,

$$
P_{O}=100 \frac{[B]^{\eta H}}{[B]^{\eta H}+\left(I C_{50}\right)^{\eta H}}
$$

where $[B]$ is the concentration of bepridil and $\eta H$ is the Hill coefficient. The best fit was obtained with $I C_{50}=1.866 \mu \mathrm{M}$ and the Hill coefficient $=-1.68$.
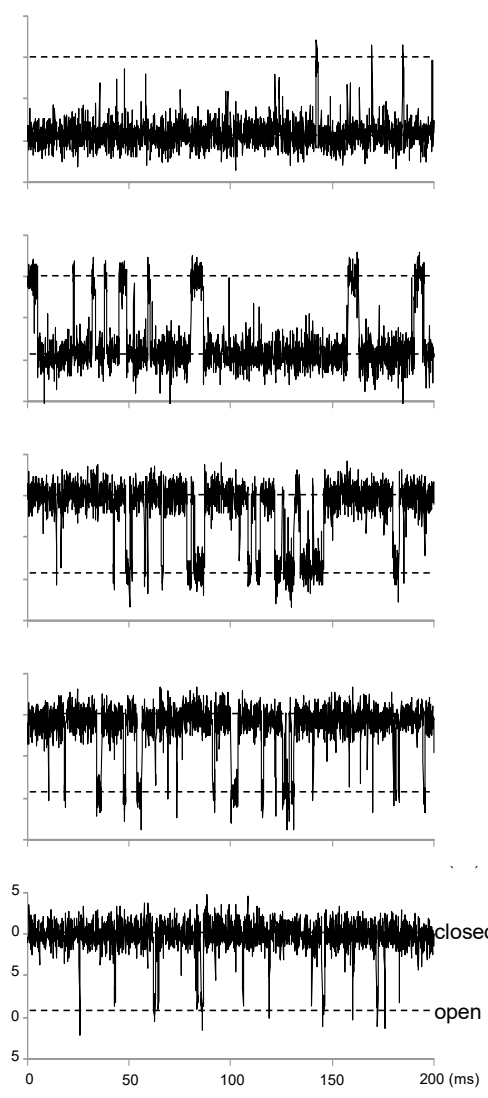

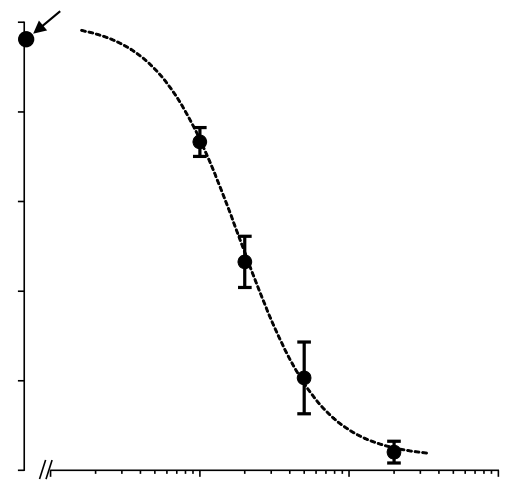

$\mathrm{P}_{\mathrm{O}}=0.02$

Peak Amp $=-8.43 \pm 0.04 \mathrm{pA}$

OT $=1.22 \pm 0.18 \mathrm{~ms}$

OdT $=2.74 \pm 0.65 \mathrm{~ms}$

$\mathrm{CT}=16.3 \pm 2.67 \mathrm{~ms}$

Fig. 2. (A) Effect of bepridil $(1-20 \mu \mathrm{M})$ on single-channel activity of a SAKca channel at $+30 \mathrm{mV}$. $P_{\mathrm{O}}$ (probability of the channel being open), OT (channel open time) and OdT (time constants for open channel dwell times) decreased, and CT (channel close time) and CdT (time constants for closed channel dwell times) increased in a dose-dependent manner. (B) $P_{0}$-bepridil concentration curve. $P_{0}$ of SAKca channel almost completely blocked with $20 \mu \mathrm{M}$ bepridil (control: $\mathrm{n}=13,1 \mu \mathrm{M}: \mathrm{n}=14,2 \mu \mathrm{M}: \mathrm{n}=8,5 \mu \mathrm{M}: \mathrm{n}=3,20 \mu \mathrm{M}: \mathrm{n}=4$ ). ${ }^{*} P<0.05$ vs. control. Data are presented as mean \pm SEM.

Figure 3 shows representative ECG and LVP traces of SIE. A QRS complex and T wave sets of SIE with patterns that differed from regular beats appeared after a short delay from initiation of the ventricular wall stretch $(\Delta \mathrm{T})$. Ectopic beats emerged without the preceding $\mathrm{P}$ wave, and usually with a compensatory pause (longer beat interval after an extrasystolic event).
The stretch induced a triangular diastolic LVP increase because of the LV volume change. This was followed by another increase in LVP from an ectopic LV contraction when SIE was initiated. The probability of SIE being caused by the LV wall stretch was $21.7 \pm 1.6 \%$, which is consistent with our previous results (Iribe et al. 2011). 


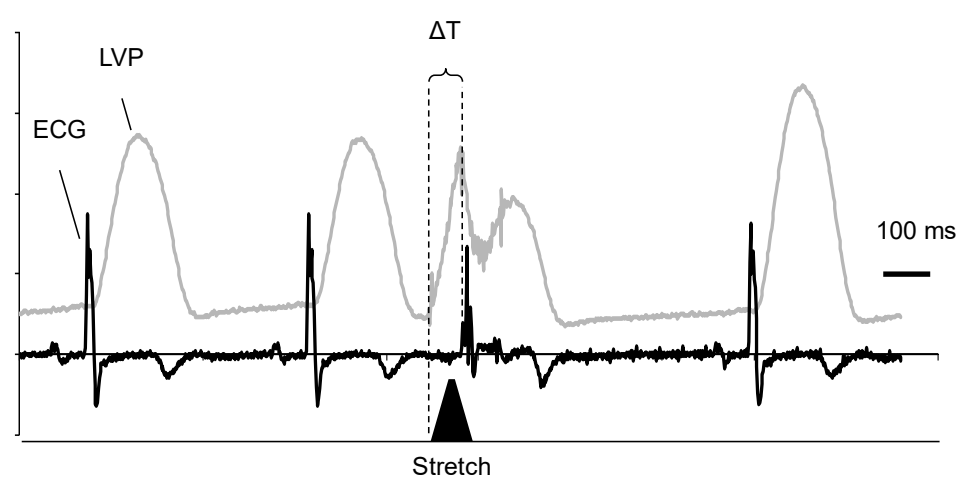

Fig. 3. Representative electrocardiogram (ECG) (black) and left volume pressure (LVP) (grey) traces in stretch-induced extrasystole (SIE). Ectopic QRS complex sets appeared on ECG after a delay $(\Delta T)$ from the beginning of the stretch signal.

We tested the effects of bepridil on SIE and compared them with those of Gadolinium $\left(\mathrm{Gd}^{3+}\right)$, a potent, but non-specific blocker of mechanosensitive channels. Figure 4 shows the effect of bepridil $(1 \mu \mathrm{M})$ and $\mathrm{GdCl}_{3}(3 \mu \mathrm{M})$ on the probability of SIE and $\Delta \mathrm{T}$ of the SIE. Panel A shows the average probabilities of SIE of all hearts (" $n$ " indicates the number of hearts analyzed). Both drugs significantly reduced the probability of SIE, however, there was no significant difference between the drugs. Then, the SIE beats with ECG traces that were clear enough to analyze $\Delta \mathrm{T}$ were selected, and the average values are shown in panel B (" $n$ " indicates the number of beats analyzed). Both drugs have a tendency to increase $\Delta \mathrm{T}$, although the increase was not statistically significant.
A

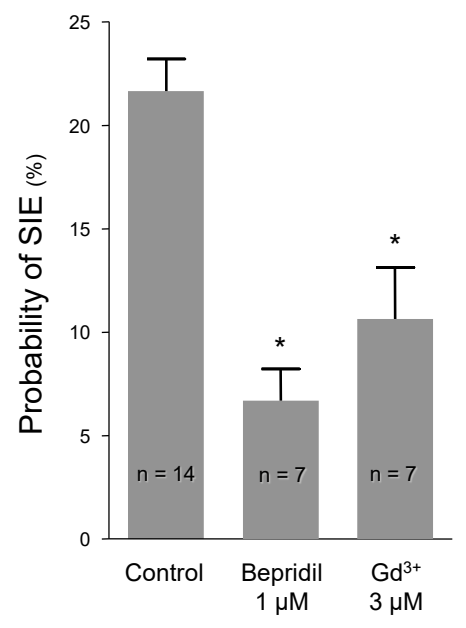

\section{Discussion}

We have previously reported that sarcolemmal stretch-activated BKca (SAKca) channels exist in post-hatch chick ventricular myocytes (Iribe et al. 2010), and we found that IbTx, a specific BKca channel blocker, significantly increase the probability of SIE, while GsMTx4, a specific $\mathrm{SAC}_{\mathrm{NS}}$ blocker, significantly decreases the probability of SIE, suggesting that the outward SAKca current counterbalances stretch-induced cation influx via $\mathrm{SAC}_{\mathrm{NS}}$ to prevent stretch-induced depolarization, namely SIE (Iribe et al. 2011).

As shown in Figure 2, the present single-channel
Fig. 4. Effects of bepridil $(1 \mu \mathrm{M})$ $(n=7)$ and $\mathrm{Gd}^{3+}(3 \mu \mathrm{M})(\mathrm{n}=10)$ on the probability of SIE and $\Delta$ T. Both drugs significantly decreased the probability of SIE and showed a tendency to increase $\Delta \mathrm{T} .{ }^{*} P<0.05$ vs. control. Data are presented as mean \pm SEM. study demonstrated that bepridil inhibits SAKca channel activity. The absolute value of the Hill coefficient (1.676) is greater than 1.0 indicating the number of bound bepridil at the maximal channel inhibition level is more than one, thus positive cooperativity among binding sites. Although a single-site inhibitor (Hill coefficient=1) requires 81 times more inhibitor concentration to achieve $90 \%$ inhibition than a $10 \%$ inhibition, Figure $2 \mathrm{~B}$ shows that Bepridil (Hill coefficient=1.686) can do the same over less than a 20 -fold concentration range.

We expected that bepridil would increase the probability of SIE by inhibiting SAKca outward current. However, contrary to our expectation, bepridil 
significantly reduced the probability of SIE in the present study, as shown in Figure 4. One possible explanation for the inconsistency between these results is that bepridil inhibits not only SAKca but also $\mathrm{SAC}_{\mathrm{NS}}$ current. As a result, the effect of blocking the outward SAKca current (facilitating SIE) can be counterbalanced and overridden by the effect of blocking the $\mathrm{SAC}_{\mathrm{NS}}$ inward current (reducing SIE). The present results show that the effect of bepridil on SIE is almost identical to that of the non-specific mechanosensitive channel blocker $\mathrm{Gd}^{3+}$ (Fig. 4). $\mathrm{Gd}^{3+}$ has been used not only as a blocker of $\mathrm{SAC}_{\mathrm{NS}}$ (Hansen et al. 1991, Yang et al. 1989) but also as a blocker of SAKca channels (Tang et al. 2003). Therefore, it is reasonable to consider that bepridil may block both $\mathrm{SAK}$ ca and $\mathrm{SAC}_{\mathrm{NS}}$.

Another finding that supports the inhibitory effect of bepridil on $\mathrm{SAC}_{\mathrm{NS}}$ is the change in $\Delta \mathrm{T}$. Although the lengthening of $\Delta \mathrm{T}$ was not significant in the present study, bepridil and $\mathrm{Gd}^{3+}$ showed the same tendency to prolong it (Fig. 4). We have previously reported that GsMTx 4 prolongs $\Delta \mathrm{T}$, and that extension of $\Delta \mathrm{T}$ is associated with slowed stretch-induced depolarization due to the decrease in stretch-induced cation influx via $\mathrm{SAC}_{\mathrm{NS}}$ (Iribe et al. 2011). Here again it is reasonable for bepridil to block $\mathrm{SAC}_{\mathrm{NS}}$ to reduce the stretch-induced cation influx (prolonging $\Delta \mathrm{T}$ ) to compensate and override the effects of the decrease in outward SAKca current (shortening $\Delta \mathrm{T}$ ).

Although the effects of bepridil on SIE suggest that it inhibits $\mathrm{SAC}_{\mathrm{NS}}$, the present study does not provide any direct evidence in electrophysiological experiments. Here again it is reasonable for bepridil to block $\mathrm{SAC}_{\mathrm{NS}}$ to reduce the stretch-induced cation influx (prolonging $\Delta \mathrm{T}$ ) to compensate and override the effects of the decrease in outward SAKca current (shortening $\Delta \mathrm{T}$ ). However, in practice, it is not feasible to perform either single-channel or whole-cell current recordings to study $\mathrm{SAC}_{\mathrm{NS}}$ in SIE.
Regarding single-channel studies, $\mathrm{SAC}_{\mathrm{NS}}$ have never been patched for single-channel current recordings in ventricular myocytes, probably because $\mathrm{SAC}_{\mathrm{NS}}$ is located in the T-tubule (Zeng et al. 2000). Therefore, examination of the effects of bepridil on a single $\mathrm{SAC}_{\mathrm{NS}}$ current is not a realistic approach. Regarding whole-cell studies, although there have been several studies of the $\mathrm{SAC}_{\mathrm{NS}}$ component in the whole-cell current (Zeng et al. 2000, Kamkin et al. 2000, Kamkin et al. 2003), all these studies were performed under static cell stretch conditions that are not suitable for the SIE study. As we previously reported, the speed of stretch significantly affects the activity of the mechanosensitive channels (Iribe et al. 2011, Iribe et al. 2010). Therefore, quick stretch needs to be applied to reproduce the same activation as in SIE. However, it is very challenging to maintain the whole-cell configuration during such a quick and dynamic cell stretch; therefore, whole-cell experiments are not a realistic option either.

In conclusion, we investigated the effects of bepridil on a single SAKca channel current in cultured chick ventricular myocytes and the effects of bepridil on SIE using isolated Langendorff-perfused chick hearts. We found that bepridil blocks SAKca channels and reduces the probability of SIE. These results suggest that bepridil possibly also blocks $\mathrm{SAC}_{\mathrm{NS}}$.

\section{Conflict of Interest}

There is no conflict of interest.

\section{Acknowledgements}

This work was supported by the Japan Society for the Promotion of Sciences (JSPS KAKENHI: 23300167, 22136008, 22240056), Daiichi Sankyo Co., Ltd and National Natural Science Foundation of China (No. 81260035).

\section{References}

BODE F, SACHS F, FRANZ MR: Tarantula peptide inhibits atrial fibrillation. Nature 409: 35-36, 2001.

COHEN CJ, SPIRES S, VAN SKIVER D: Block of t-type ca channels in guinea pig atrial cells by antiarrhythmic agents and ca channels antagonists. J Gen Physiol 100: 703-728, 1992.

COSNIER D, DUCHENNE-MARULLAZ P, RISPAT G, STREICHENBERGER G: Cardiovascular pharmacology of bepridil (1[3 isobutoxy 2 (benzylphenyl) amino] propyl pyrrolidine hydrochloride) a new potential anti-anginal compound. Arch Int Pharmacodyn Ther 225: 133-151, 1977.

GILL A, FLAIM SF, DAMIANO BP, SIT SP, BRANNAN MD: Pharmacology of bepridil. Am J Cardiol 69: 11D-16D, 1992. 
HANSEN DE, BORGANELLI M, STACY GP JR, TAYLOR LK: Dose-dependent inhibition of stretch-induced arrhythmias by gadolinium in isolated canine ventricles. Evidence for a unique mode of antiarrhythmic action. Circ Res 69: 820-831, 1991.

HALL CE, HURTADO R, HEWETT KW, SHULIMOVICH M, POMA CP, RECKOVA M, JUSTUS C, PENNISI DJ, TOBITA K, SEDMERA D, GOURDIE RG, MIKAWA T: Hemodynamic-dependent patterning of endothelin converting enzyme 1 expression and differentiation of impulse-conducting Purkinje fibers in the embryonic heart. Development 131: 581-592, 2004.

IIJIMA K, CHINUSHI M, IZUMI D, AHARA S, FURUSHIMA H, KOMURA S, HOSAKA Y, SANADA A, SATO A, AIZAWA Y: Effect of bepridil in atrial fibrillation inducibility facilitated by vagal stimulation.-prevention of vagal nerve activation-induced shortening of the atrial action potential duration. Circ J 74: 895-902, 2010.

IRIBE G, JIN H, NARUSE K: Role of Sarcolemmal BKca channels in stretch-induced extrasystoles in isolated chick hearts. Circ J 75: 2552-2558, 2011.

IRIBE G, JIN H, KAIHARA K, NARUSE K: Effects of axial stretch on sarcolemmal BKca channels in post-hatch chick ventricular myocytes. Exp Physiol 95: 699-711, 2010.

KAMKIN A, KISELEVA I, ISENBERG G: Stretch-activated currents in ventricular myocytes: Amplitude and arrhythmogenic effects increase with hypertrophy. Cardiovasc Res 48: 409-420, 2000.

KAMKIN A, KISELEVA I, ISENBERG G: Ion selectivity of stretch-activated cation currents in mouse ventricular myocytes. Pflugers Arch 446: 220-231, 2003.

KAWAKUBO T, NARUSE K, MATSUBARA T, HOTTA N, SOKABE M: Characterization of a newly found stretchactivated $\mathrm{K}_{\mathrm{Ca}, \mathrm{ATP}}$ channel in cultured chick ventricular myocytes. Am J Physiol 276: H1827-H1838, 1999.

KOHL P, HUNTER P, NOBLE D: Stretch-induced changes in heart rate and rhythm: Clinical observation, experiments and mathematical models. Prog Biophys Mol Biol 71: 91-138, 1999.

LAB MJ, TAGGART P, SACHS F: Mechano-electric feedback. Cardiovasc Res 32: 1-2, 1996.

LIM-LEVY F, BABLER SA, DE GROOT-KOSOLCHAROEN J, KOSOLCHAROEN P, KRONCKE GM: Is milking and stripping chest tubes really necessary? Ann Thorac Surg 42: 77-80, 1986.

NARUSE K, TANG QY, SOKABE M: Stress-axis regulated exon (strex) in the c terminus of BKca channels is responsible for stretch sensitivity. Biochem Biophys Res Commun 385: 634-639, 2009.

NESBITT AD, COOPER PJ, KOHL P: Rediscovering commotion cordis. Lancet 357: 1195-1197, 2001.

OWEN JD: The determination of the stability constant for calcium-EGTA. Biochim Biophys Acta 45: 321-325, 1976.

PELLIS T, KOHL P: Extracorporeal cardiac mechanical stimulation: Precordial thump and precordial percussion. $B r$ Med Bull 93: 161-177, 2010.

SINGH BN: Comparative efficacy and safety of bepridil and diltiazem in chronic stable angina pectoris refractory to diltiazem. The bepridil collaborative study group. Am J Cardiol 68: 306-312, 1991.

TANG QY, QI Z, NARUSE K, SOKABE M: Characterization of a functionally expressed stretch-activated BKca channels cloned from chick ventricular myocytes. J Membr Biol 196: 185-200, 2003.

VOUKYDIS PC, COHEN SI: Catheter-induced arrhythmias. Am Heart J 88: 588-592, 1974.

WANG JC, KIYOSUE T, KIRIYAMA K, ARITA M: Bepridil differentially inhibits two delayed rectifier $\mathrm{K}^{+}$currents, $\mathrm{I}_{\mathrm{kr}}$ and $\mathrm{I}_{\mathrm{ks}}$, in guinea-pig ventricular myocytes. Br J Pharmacol 128: 1733-1738, 1999.

YAMASHITA T, OGAWA S, SATO T, AIZAWA Y, ATARASHI H, FUJIKI A, INOUE H, ITO M, KATOH T, KOBAYASHI Y, ET AL.: Dose-response effects of bepridil in patients with persistent atrial fibrillation monitored with transtelephonic electrocardiograms: A multicenter, randomized, placebo-controlled, double-blind study (j-baf study). Circ J 73: 1020-1027, 2009.

YANG XC, SACHS F: Block of stretch-activated ion channels in xenopus oocytes by gadolinium calcium ions. Sciences 243: 1068-1071, 1989.

ZENG T, BETT GC, SACHS F: Stretch-activated whole cell currents in adult rat cardiac myocytes. Am J Physiol Heart Circ Physiol 278: H548-H557, 2000. 